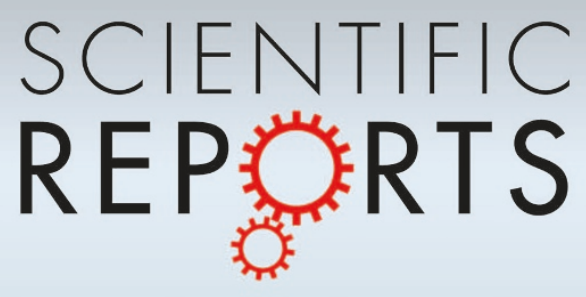

\section{OPEN}

SUBJECT AREAS:

COMPLEX NETWORKS

NONLINEAR PHENOMENA

Received

27 September 2013

Accepted

6 November 2013

Published

21 November 2013

Correspondence and requests for materials should be addressed to

N.A.M.A. (nuno@ ethz.ch)

\title{
Breathing synchronization in interconnected networks
}

\author{
V. H. P. Louzada', N. A. M. Araújo', J. S. Andrade, Jr. ${ }^{1,2}$ \& H. J. Herrmann ${ }^{1,2}$
}

${ }^{1}$ Computational Physics, IfB, ETH-Honggerberg, Wolfgang-Pauli-Strasse 27, 8093 Zurich, Switzerland, ${ }^{2}$ Departamento de Física, Universidade Federal do Ceará, 6045 1-970 Fortaleza, Ceará, Brazil.

Global synchronization in a complex network of oscillators emerges from the interplay between its topology and the dynamics of the pairwise interactions among its numerous components. When oscillators are spatially separated, however, a time delay appears in the interaction which might obstruct synchronization. Here we study the synchronization properties of interconnected networks of oscillators with a time delay between networks and analyze the dynamics as a function of the couplings and communication lag. We discover a new breathing synchronization regime, where two groups appear in each network synchronized at different frequencies. Each group has a counterpart in the opposite network, one group is in phase and the other in anti-phase with their counterpart. For strong couplings, instead, networks are internally synchronized but a phase shift between them might occur. The implications of our findings on several socio-technical and biological systems are discussed.

- echnology has furnished us with global connectivity changing the functioning of cooperative work, international business, and interpersonal relationships. For example, despite the ever faster Internet connections, there will always be a physical limit speed to information transport, thereby imposing a time delay in communication. As we discuss here, this time delay might pose a real challenge to the synchronizability of oscillators. Therefore, understanding the consequences of a communication lag is of major concern in different fields ${ }^{1-3}$. For example, the plasmodium Physarum polycephalum, an amoeba-like organism consisting of a network of tubular structures for protoplasm flow, naturally shows periodic variations in its thickness, a necessary feature for its survival. A controlled setup has been prepared by Takamatsu et al. where two regions of the same organism have been physically separated by a certain distance with the possibility of fine tuning the communication between them ${ }^{4,5}$. Depending on the coupling strength and time delay, the two regions have been shown to present phase and anti-phase synchronization of the oscillatory thickness. This is precisely what we find in the regime of strong intra-network coupling. As discussed in the final section, this biological system might be a prototype to experimentally evaluate the different regimes reported here. In what follows, we discuss the general case of two interconnected networks but our study might have impact on several biological and techno-social systems as, for example, functional brain networks, living oscillators, or coupled power grids, as discussed at the end of this paper.

Recent geometrical studies of coupled networks with intra- and inter-network links have revealed novel features never observed for isolated networks ${ }^{6}$. In particular, it has been shown that the overall robustness is reduced $^{7-11}$ and the collapse of the system occurs through large cascades of failures ${ }^{12,13}$. Dynamic properties of coupled networks have also been studied ${ }^{14-21}$, but the impact of a time delay on their synchronization is still an open issue, which we will address here. Typically, the intra- and inter-network couplings have different time scales. For simplicity, we consider the case where intra-network interactions can be considered instantaneous and the inter-network ones have a communication lag that depends on the distance between networks. In particular, we show that, when isolated, the two networks would naturally move in unison. However, when interacting the oscillators in the same network split into two groups, synchronized with different frequencies, leading to breathing synchronization.

The Kuramoto model is the standard theoretical framework for studying synchronizability of networks ${ }^{22-37}$. A population $\Theta$ of $n$ Kuramoto oscillators is considered to be mutually interacting. We consider a random graph of average degree four. Each oscillator $i \in \Theta$ is described by a phase $\theta_{i}(t)$, representing its current position, and a natural frequency $\omega_{i}$. For simplicity, we assume the same frequency $\omega_{i} \equiv \omega_{0}$ for all oscillators. The actual frequency of an oscillator is defined as the time derivative of the phase, $\dot{\theta}_{i}(t)$. To move harmoniously, oscillators try to synchronize their frequencies and phases. This interaction can be modeled in terms of the Kuramoto model 
as $\dot{\theta}_{i}=\omega_{0}+\sigma \sum_{j=1}^{n} A_{i j}^{\Theta} \sin \left(\theta_{j}-\theta_{i}\right)$, where the sum goes over all other oscillators $(i \neq j), \sigma$ is the coupling strength between them, and $\mathrm{A}^{\boldsymbol{\Theta}}$ is the connectivity matrix such that $A_{i j}^{\Theta}=1$ if oscillator $i$ is influenced by $j$ and zero otherwise. We also assume that all oscillators have the same unitary amplitude, so that the state of each one can be described by a phasor $e^{i \theta_{i}(t)}$.

The collective motion, namely, the synchronization of the network, is characterized here by the complex order parameter $r_{\Theta}(t) e^{i \Psi(t)}=\frac{1}{n} \sum_{j=1}^{n} e^{i \theta_{j}(t)}$, where the sum goes over all oscillators, $\Psi(t)$ is the average phase, and the amplitude $0 \leq\left|r_{\Theta}(t)\right| \leq 1$ measures the global coherence, i.e., how synchronized the oscillators are. If $r_{\Theta}(t)=1$ all oscillators are synchronized, while very low values of $r_{\Theta}$ imply that a significant fraction of oscillators are out of phase.

We introduce now a second population $\Gamma \neq \Theta$, also of $n$ oscillators interacting in a random graph of average degree four, representing the second network. We couple each $j \in \Gamma$ with one, and only one, corresponding partner $i \in \Theta$, forming the inter-network couplings. In analogy to oscillators in $\Theta$, the motion of each oscillator is described by a phasor $e^{i \gamma_{j}(t)}$, of phase $\gamma_{j}(t)$. The inter-network coupling is subjected to a time delay $\tau$, corresponding to the time required for information to travel between networks ${ }^{38}$. Previous studies introduced time delay among oscillators of the same population ${ }^{39,40}$. Here we consider the competition between an instantaneous intra-network and a delayed inter-network coupling. In a nutshell, the dynamics of oscillators is described by,

$$
\left\{\begin{array}{l}
\dot{\theta}_{i}=\omega_{0}+\sigma_{\mathrm{EX}} \sin \left(\gamma_{j(i)}^{t-\tau}-\theta_{i}\right)+\sigma_{\mathrm{IN}} \sum_{k=1}^{N} A_{i k}^{\Theta} \sin \left(\theta_{k}-\theta_{i}\right) \\
\dot{\gamma}_{j}=\omega_{0}+\sigma_{\mathrm{EX}} \sin \left(\theta_{i(j)}^{t-\tau}-\gamma_{j}\right)+\sigma_{\mathrm{IN}} \sum_{k=1}^{N} A_{i k}^{\Gamma} \sin \left(\gamma_{k}-\gamma_{j}\right)
\end{array},\right.
$$

where the superscript $t-\tau$ indicates the instant when the phases are calculated, and $\sigma_{\mathrm{EX}}$ and $\sigma_{\mathrm{IN}}$ are the inter and intra-network couplings, respectively.

\section{Results}

We observe that for two interconnected networks of oscillators with time delay, a weak intra-network coupling, and random initial distribution of phases, two frequency communities emerge within the same network, each synchronized with its mirror in a breathing mode, as shown in Fig. 1(a). In the figure, the color describes the frequency and the vertical position the phase. The frequency synchronization within groups occurs with phase locking. Interestingly, inter-network coupled pairs of nodes oscillate with the same frequency (same color) but might be either in phase or anti-phase (phase shift of $\pi$ ). Consequently, the presence of these two frequency groups affects the perception of the new global oscillatory state, which we call breathing synchronization. Figure $1(\mathrm{~b})$ shows the time evolution of the order parameters $r_{\Theta}$ and $r_{\Gamma}$ for each population, quantifying this breathing behavior. For each curve, the maximum corresponds to the instant at which both groups of frequencies are in phase, while the minimum to an anti-phase between groups in the same network. Additionally, since for one frequency there is a phase shift of $\pi$ between inter-network pairs of nodes, the minimum in one network corresponds, necessarily, to the maximum in the other. Cohesion within each community affects the amplitude of the breathing, as indicated by the order parameters for different values of $\sigma_{\text {IN }}$ in Fig. 1(b). The weaker the intra-network coupling, the smaller is this amplitude.

The observed breathing behavior is in deep contrast with what is expected for an isolated network $\left(\sigma_{\mathrm{EX}}=0\right)$. For isolated networks, the classical Kuramoto model is recovered, with frequency and phase synchronization emerging at a critical coupling $\sigma_{\mathrm{IN}}=\sigma_{\mathrm{IN}}^{*}$. Above this threshold, a macroscopic fraction of oscillators is synchronized, all

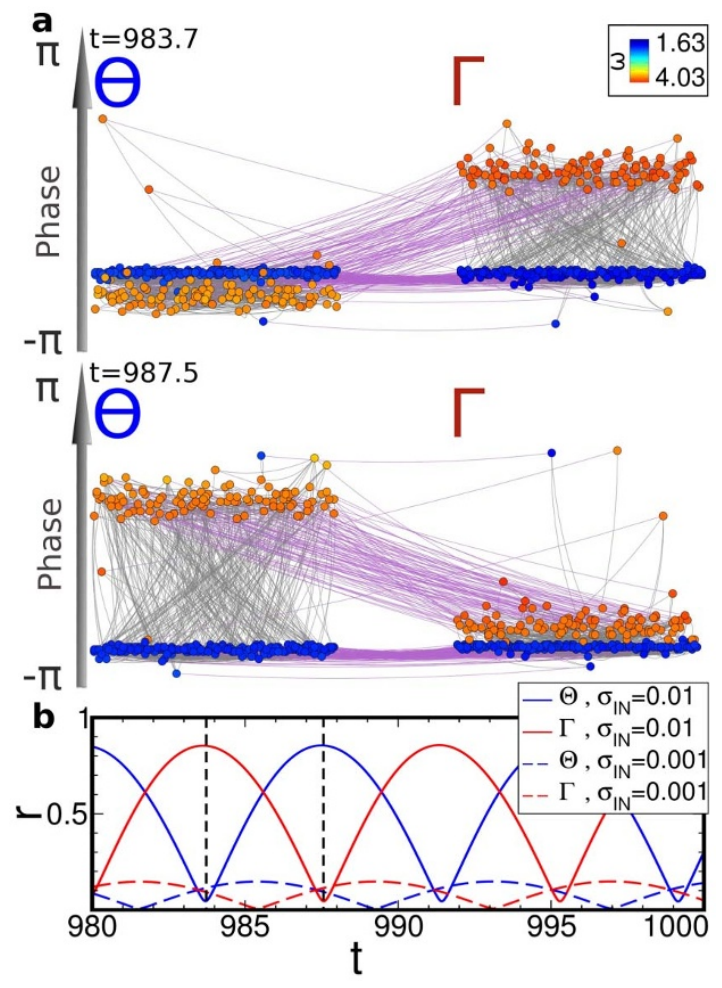

Figure 1 | The interactions between a strongly delayed inter-network coupling and a weak intra-network coupling create two communities of different frequencies in steady state. (a), Snapshot of populations at two different time steps (black dashed vertical lines in $\mathrm{b}$ ) near the steady state, for $\omega_{0}=1.0, \tau=1.53, \sigma_{\mathrm{IN}}=0.01$, and $\sigma_{\mathrm{EX}}=0.5$. The vertical position of each oscillator represents its phase, from $-\pi$ to $\pi$, and the color represents the frequencies achieved with oscillators mostly presenting values near the theoretical frequencies (1.63 and 4.63) of the steady state. Superposition of these two communities leads to breathing synchronization. (b), Time evolution of the order-parameter of populations $\Theta$ (blue) and $\Gamma$ (red) composed of $n=305$ oscillators each with $\omega_{0}=1.0, \tau=1.53$, and $\sigma_{\mathrm{EX}}=$ 0.5 . Two scenarios of weak intra-network coupling are represented: $\sigma_{\mathrm{IN}}=$ 0.01 (continuous lines) and $\sigma_{\mathrm{IN}}=0.001$ (dashed lines).

with the same frequency and phase. The value of $\sigma_{\mathrm{IN}}^{*}$ increases with the variance of the natural frequency distribution. Since here we consider the same natural frequency for all oscillators $\left(\omega_{i} \equiv \omega_{0}\right)$, $\sigma_{\mathrm{IN}}^{*} \rightarrow 0$. The group of synchronized oscillators has frequency $\omega=\omega_{0}$ and the order parameter $r_{\Theta}(t)$ (or $\left.r_{\Gamma}(t)\right)$ saturates in time at a nonzero steady-state value ${ }^{22}$, which is a monotonically increasing function of $\left(\sigma_{\mathrm{IN}}-\sigma_{\mathrm{IN}}^{*}\right)$. Interestingly, in the case of coupled networks, and for sufficient inter-network couplings, none of the two frequencies is $\omega_{0}$.

To better understand the breathing synchronization, and in particular the emergence of frequency groups, let us consider the case of two coupled oscillators with time delay. The analytic solution obtained by Schuster and Wagner ${ }^{38}$ for this problem indicates that, depending on the initial phase difference between oscillators (see Fig. S1 in the Supplemental Material), the pair can synchronize with different frequencies $\omega$, which are solutions of,

$$
\omega=\omega_{0}-\sigma_{\mathrm{EX}} \sin (\omega \tau) .
$$

In spite of oscillating with the same frequency in the stationary state, the two oscillators might either be in phase, if $\cos (\omega \tau)>0$, or antiphase, otherwise. In the case of interconnected networks, in the limit $\sigma_{\mathrm{IN}}=0$, the stationary state is expected to include all possible solutions of Eq. 2. Surprisingly, our results with a weak coupling reveal instead two frequency groups with phase locking. Nevertheless, the 


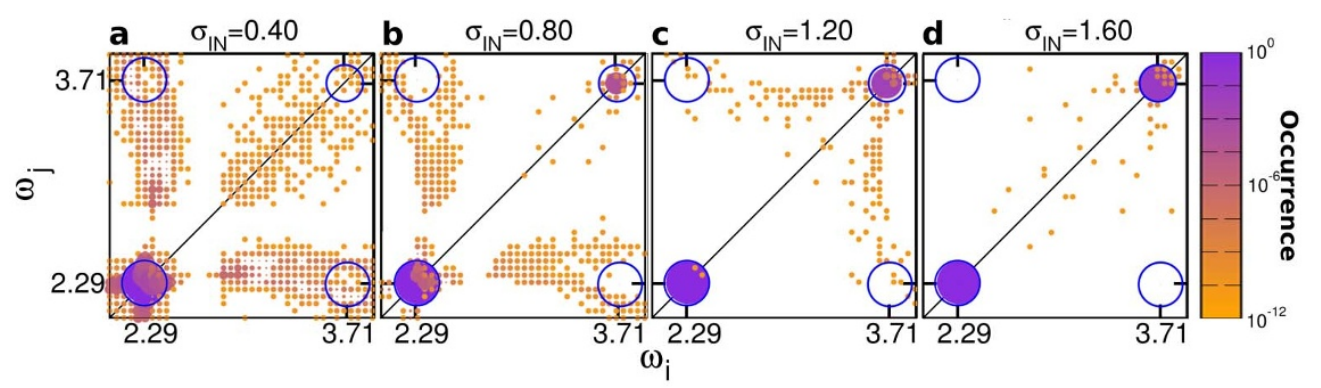

Figure $2 \mid$ Scatter plot for the matrix of frequency pairs of intra-network neighboring oscillators, for 500 different realizations of random coupled networks of $n=750$, with $\omega_{0}=2.75, \tau=1.53, \sigma_{\mathrm{EX}}=1.5$, and various $\sigma_{\mathrm{IN}}$, namely, 0.4 (a), 0.8 (b), 1.2 (c), and 1.6 (d). Empty and filled circles are centered on the frequency pairs $\left(\omega_{i}, \omega_{j}\right)$ for each $(i, j)$ neighboring nodes within a network, calculated with a $2 \mathrm{D}$ binning of size 0.05 . The filled circles color, according to a purple-yellow scale, corresponds to their relative occurrence in the dataset: purple circles are the predominant frequencies registered, while yellow circles are less common. For comparison, blue empty circles correspond to results for $\sigma_{\mathrm{IN}}=0$. The size of the symbols is also used for the relative occurrence of each pair.

observed frequencies are consistent with the solution of Eq. 2 and are unique with respect to $\omega_{0}$ and $\tau$. The final frequency of a pair of oscillators only depends of their relative initial displacement.

As we show next, when the internal coupling $\left(\sigma_{\mathrm{IN}}\right)$ is further increased, breathing synchronization is no longer stable and each network is synchronized, in one of two other synchronization regimes. In simulations with $\sigma_{\mathrm{EX}}=1.5, \omega_{0}=2.75$ and $\tau=1.53$, when $\sigma_{\mathrm{IN}}=0.4$ interactions among oscillators in the same network become more relevant than the inter-network delayed coupling, and the larger frequency group, in terms of size, dominates over the smaller one. This competition results in all oscillators synchronizing at the same frequency and the order parameter of each network saturates in time. To systematically study the dependence on $\sigma_{\mathrm{IN}}$, we analyze the frequency correlation among intra-network neighbors $i$ and $j$. Figure 2 shows the scatter plots of the pair $\left(\omega_{i}, \omega_{j}\right)$ for different values of intra-network coupling strengths. The limit $\sigma_{\mathrm{IN}}=$ 0 is represented by the blue empty circles in all panels and the radius corresponds to the relative population of pairs when considering several samples. In this limit, the oscillators have all one of two possible frequencies, with four possible combinations of frequency pairs. From the relative size of the circles, we observe that the lowest frequency ( $\omega \approx 2.3$ for $\omega_{0}=2.75$ and $\tau=1.53$ ) is the most populated one. As shown in Fig. 2(a), for $\sigma_{\mathrm{IN}}=0.4$ most nodes are synchronized with the lowest frequency and therefore a large percentage of the pairs are in the left-bottom corner. Similarly to the Kuramoto model, in this competing state, oscillators synchronize at a unique stable frequency $(\omega \approx 2.3)$, which is a solution of Eq. 2 . As $\sigma_{\text {IN }}$ is further increased (Fig. 2(b)-(d)), due to the strength of the intranetwork coupling, each network tends to behave like a supernode and, depending on the initial conditions, one of two frequencies is obtained, which is again a solution of Eq. 2. Further analysis across samples (See Fig. S2 in Supplemental Material) also shows that the average phase displacement between pairs of oscillators in different networks reaches $\Delta=\pi$ for intermediary values of $\sigma_{\mathrm{IN}}$, and decreases again once the supernodes are formed (See Fig. S2(a) in Supplemental Material). For large $\sigma_{\mathrm{IN}}$, the supernodes can be either in phase or anti-phase and, therefore, the average variance within a network has a value between zero and $\pi$ (See Fig. S2(b) in Supplemental Material). Results are qualitatively similar for networks with fixed node degree (See Fig. S2(c-d) in Supplemental Material) or with different average degree (See Fig. S2(e-f) in Supplemental Material).

To summarize the effect of several combinations of parameters, we plot in Fig. 3 the phase diagram in the space of the two coupling strengths $\left(\sigma_{\mathrm{IN}}\right.$ and $\left.\sigma_{\mathrm{EX}}\right)$. To identify each regime, we compute the amount of oscillators with steady frequency below and above the mean value of possible frequencies (see Section Methods), $A_{1}$ and $A_{2}$, respectively, over different samples (see top inset of Fig. 3). The color map of the main plot of Fig. 3 shows the ratio of these quantities. While the blue area represents the domain of $\sigma_{\mathrm{IN}}$ and $\sigma_{\mathrm{EX}}$ combinations that leads to the smaller frequency, the shades in red represent the two regions where two frequencies can be achieved. Note that, the nature of the two synchronization regimes in red is different. The one in the left (lower $\sigma_{\mathrm{IN}}$ ) is characterized by the breathing behavior due to the presence of two frequency groups within each network. By contrast, in the supernode regime all nodes within a network are in phase locking, with the same frequency and, therefore, the order parameter is constant in time in the steady state. In the bottom inset, we show the phase boundaries for different time delays. From this, one can also see that the transition between regimes changes substantially for different time delays. Since delay and natural frequency are not multiples, harmonic interactions are considered negligible. Table I contains a brief summary of all states

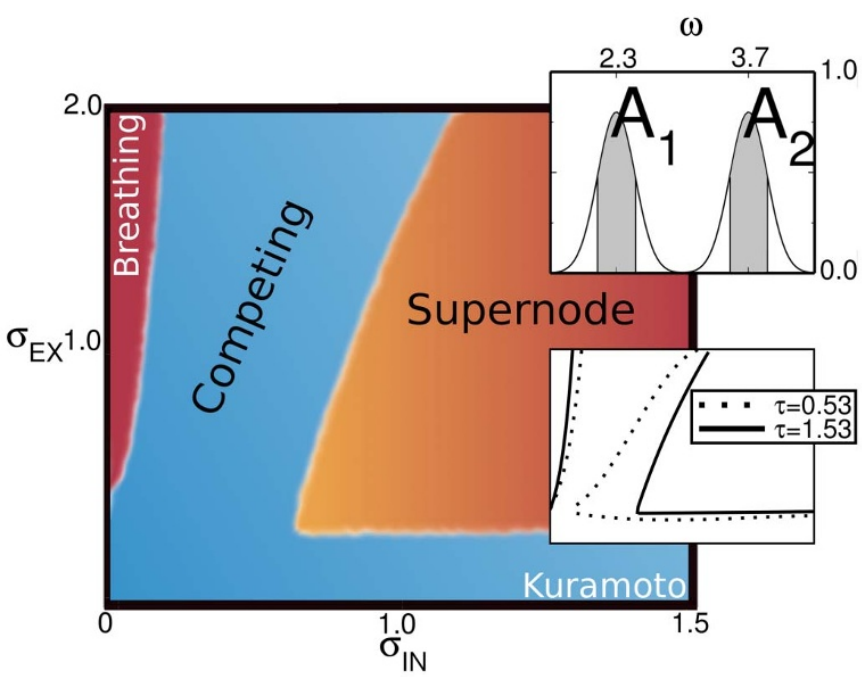

Figure 3 Phase diagram for delayed coupled networks. Parameter space of two coupling strengths $\sigma_{\mathrm{EX}}$ and $\sigma_{\mathrm{IN}}$ showing that the prevalence of one frequency over the other changes according to the coupling strengths. The color of each region represents the occurrence of the two theoretical frequencies: red if two frequencies $(\omega=2.3$ and $\omega=3.7)$ and blue if only one $(\omega=2.3)$ is observed. Shaded regions mark the boundaries between states. Top inset is an example of the histogram used to calculate the main panel: areas around the theoretical frequencies are defined $\left(A_{1}\right.$ and $\left.A_{2}\right)$ and their ratio used to define the prevalence of only one or two of them. The lower inset exhibits the state boundaries for different time delays. The dominant mechanisms of each region are labeled accordingly: Breathing, Kuramoto $^{22}$, Competing, and Supernode states. Regions are defined based on simulations over 300 different realizations of random coupled networks of $n=500$, with $\omega_{0}=2.75$ and $\tau=1.53$. 
Table I | Summary of different synchronization regimes. A brief description of the properties of all states encountered for $\omega_{0}=2.75$ and $\tau=$ 1.53

State

Breathing

Competing

Supernode
Frequency groups

Two groups: $\omega=2.29$ (low freq.) and $\omega=3.71$ (high freq.)

One group: $\omega=2.29$

One group: $\omega=2.29$ or $\omega=3.71$
Phase shift

$\pi$ for low freq. and 0 for high freq.

$\pi$

$\pi$ if low freq. or 0 if high freq. reported in Fig. 3. In Fig. S3 of the Supplemental Material we show that the transitions between regimes with one and two stable frequencies are abrupt.

\section{Discussion}

The presence of a time delay between two coupled networks of oscillators poses a new challenge to the global control of the system. We have shown that the interplay between coupling and delay leads to states of either a unique or two possible synchronized frequencies. We have found that, even with a weak intra-network coupling, oscillators within the same network split into two frequency groups. Each group has a mirror one in the other network oscillating at the same frequency. However, depending on their frequency, a group can be either in phase or anti-phase with its mirror in the other network, resulting in breathing synchronization. Also, we show that an arbitrary increase of the intra-network coupling is not an option to achieve phase and frequency synchronization regardless of its initial conditions. In a certain region of the parameter space, the intranetwork coupling promotes the formation of two supernodes (one per network), and two frequencies become stable. We have numerically identified the transition regions between regimes. Future works should consider recent advances on group synchronization to analytically study these transitions through linear stability analysis using the master stability function ${ }^{41,42}$.

As previously mentioned, it is possible to prepare controlled experiments to evaluate the existence of these different regimes in biological systems. Takamastu et al. ${ }^{4}$ have shown that the distance and interaction strength between regions of a plasmodial slime mold can be fine tuned. This organism is a network of tubular structures with periodic variations in the thickness. In the experimental study, the focus was only on the regime where the intra-region interaction is much stronger than the inter-region one. Using the same methodology, it is possible to control the intra-region interaction and study the different regimes described here. In particular, it would be of interest to observe oscillations with two different frequencies within the same region due to the communication lag with the other region, resulting in breathing synchronization.

Another example where synchronization in interdependent networks certainly plays a relevant role is the human brain. Being a highly modular structure, its coherent operation must rely on the independence of different brain modules, which are functionally specialized, as well as on their efficient connection to ensure proper information transmission and processing. In a recent study ${ }^{43}$, it was shown that the optimal integration of these modules, which can be interpreted as complex networks made of intra-network couplings, is achieved through the addition of long-range inter-network ties, therefore behaving globally as a small-world system. Moreover, their experimental observations are also consistent with the fact that these inter-network couplings should be spatially organized in such a way as to maximize information transfer under wiring cost constraints $^{44,45}$. To accomplish multisensory integration in this intricate architecture of neuronal firing-oscillators ${ }^{46}$, however, information originating from distinct sensory modalities (vision, audition, taction, etc.) must ultimately be processed in a synchronized way. This is typically the case when the processing of a visual signal influences the perception of an auditory stimulus and vice-versa ${ }^{47,48}$.

\section{Methods}

Equation 1 has been numerically solved using a fourth order Runge-Kutta method with discrete time steps $\delta t=0.003$. The stable frequencies were computed at $t_{\max }=$ 100 , using the difference between phases after one $\delta t$ step. The natural frequency has been chosen as $\omega_{0}=1.00$ in Fig. 1 and $\omega_{0}=2.75$ for Figs. 2-3. Initial phases of oscillators in all simulations have been sampled from a random uniform distribution between $-\pi$ and $\pi$. Different values of $\omega_{0}$ do not affect qualitatively the results. The same values of $\delta t$ and $t_{\max }$ were adopted for all simulations in this study.

In Fig. 1, Panels a) and b) are based on one pair of undirected random networks of average degree four and 305 nodes in each. Oscillators in this figure have been simulated for $\tau=1.53, \sigma_{\mathrm{EX}}=1.5$. Panel a) is based on $\sigma_{\mathrm{IN}}=0.01$.

In Fig. 2, Panels a)-d) contain the simultaneous representation of 500 pairs of random networks of 750 nodes. Color and size of each point represents the relative occurrence in all data. Oscillators in this figure have been simulated for $\tau=1.53$ and $\sigma_{\mathrm{EX}}=1.5$.

Fig. 3 is a schematic representation based on the average over 300 pairs of undirected random networks of average degree four and 500 nodes in each. The upper inset is a graphical representation of the histogram of all stable frequencies. The lower inset contains the same study for different delays, also averaged over 300 pairs of undirected random networks of average degree 4 and 500 nodes in each. A cutoff of $\omega=$ 3.00 , the midpoint of the stable frequencies for $\sigma_{\mathrm{IN}}=0$, was used to determine the areas $A_{1}$ and $A_{2}$. Colors in the main panel are defined according to the ratio of A1 and A2: blue if $\log \left(A_{1} / A_{2}\right)<4$ and red if $\log \left(A_{1} / A_{2}\right)>4$, with shades of these colors used to represent the transition regions. To avoid the effect of oscillators that did not reach a stable state by the end of the simulation, we consider only frequencies with a relative occurrence of more than $10 \%$. Oscillators in this figure have been simulated with $\tau=$ 1.53 in the main panel and $\tau=0.53$ in the lower panel.

1. Duke, C. Prosperity, complexity and science. Nat. Phys. 2, 426-428 (2006).

2. Boccaletti, S., Latora, V., Moreno, Y., Chavez, M. \& Hwang, D. Complex networks: Structure and dynamics. Phys. Rep. 424, 175-308 (2006).

3. Helbing, D. Systemic Risks in Society and Economics. Paper prepared for IRGC Workshop on Emerging Risks, (2009).

4. Takamatsu, A., Fujii, T. \& Endo, I. Time delay effect in a living coupled oscillator system with the plasmodium of Physarum polycephalum. Phys. Rev. Lett. 85, 2026 (2000).

5. Takamatsu, A., Takaba, E. \& Takizawa, G. Environment-dependent morphology in plasmodium of true slime mold Physarum polycephalum and a network growth model. J. Theor. Biol. 256, 29-44 (2009).

6. Gao, J., Buldyrev, S. V., Stanley, H. E. \& Havlin, S. Networks formed from interdependent networks. Nat. Phys. 8, 40-48 (2011).

7. Schneider, C. M., Moreira, A. A., Andrade, J. S., Havlin, S. \& Herrmann, H. J. Mitigation of malicious attacks on networks. Proc. Nat. Acad. USA 108, 3838-3841 (2011).

8. Schneider, C. M., Yazdani, N., Araujo, N. A. M., Havlin, S. \& Herrmann, H. J. Towards designing robust coupled networks. Sci. Rep. 3, 1969 (2013).

9. Herrmann, H. J., Schneider, C. M., Moreira, A. A., Andrade, J. S. Jr. \& Havlin, S. Onion-like network topology enhances robustness against malicious attacks. J. Stat. Mech. P01027 (2011).

10. Schneider, C. M., Araujo, N. A. M. \& Herrmann, H. J. Algorithm to determine the percolation largest component in interconnected networks. Phys. Rev. E 87, 043302 (2013).

11. Louzada, V. H. P., Daolio, F., Herrmann, H. J. \& Tomassini, M. Smart rewiring for network robustness. J. Complex Netw.; DOI:10.1093/comnet/cnt010 (2013).

12. Buldyrev, S. V., Parshani, R., Paul, G., Stanley, H. E. \& Havlin, S. Catastrophic cascade of failures in interdependent networks. Nature 464, 1025-1028 (2010).

13. Brummitt, C. D., D’Souza, R. M. \& Leicht, E. A. Suppressing cascades of load in interdependent networks. Proc. Nat. Acad. USA 109, E680-E689 (2011).

14. Li, C., Sun, W. \& Kurths, J. Synchronization between two coupled complex networks. Phys. Rev. E 76, 046204 (2007).

15. Sorrentino, F. \& Ott, E. Network synchronization of groups. Phys. Rev. E 76, 056114 (2007).

16. Wu, X., Zheng, W. X. \& Zhou, J. Generalized outer synchronization between complex dynamical networks. Chaos 19, 013109 (2009).

17. Shang, Y., Chen, M. \& Kurths, J. Generalized synchronization of complex networks. Phys. Rev. E 80, 027201 (2009).

18. Mao, X. Stability switches, bifurcation, and multi-stability of coupled networks with time delays. Appl. Math. Comput. 218, 6263-6274 (2012). 
19. Araújo, N. A. M., Seybold, H., Baram, R. M., Herrmann, H. J. \& Andrade, J. S. Optimal Synchronizability of Bearings. Phys. Rev. Lett. 110, 064106 (2013).

20. Cardillo, A. et al. Emergence of network features from multiplexity. Sci. Rep. $\mathbf{3}$, 1344 (2013).

21. Gómez, S. et al. Diffusion Dynamics on Multiplex Networks. Phys. Rev. Lett. 110, 028701 (2013)

22. Kuramoto, Y. \& Nishikawa, I. Statistical Macrodynamics of Large Dynamical Systems. Case of a Phase Transition in Oscillator Communities. J. Stat. Phys. 49 569-605 (1987).

23. Néda, Z., Ravasz, E., Vicsek, T., Brechet, Y. \& Barabási, A. L. Physics of the rhythmic applause. Phys. Rev. E 61, 6987-6992 (2000).

24. Boccaletti, S., Kurths, J., Osipov, G., Valladares, D. \& Zhou, C. The synchronization of chaotic systems. Phys. Rep. 366, 1-101 (2002).

25. Wang, X. F. Complex Networks: Topology, Dynamics and Synchronization. Int. J. Bifucart. Chaos 12, 885-916 (2002).

26. Pikovsky, A., Rosenblum, M. \& Kurths, J. Synchronization: A universal concept in non-linear sciences. (Cambridge Univ. Press, Cambridge, 2003).

27. Strogatz, S. H. Sync: The Emerging Science of Spontaneous Order. (Hyperion, New York, 2003).

28. Li, C. \& Chen, G. Synchronization in general complex dynamical networks with coupling delays. Physica A 343, 263-278 (2004).

29. Lü, J. \& Chen, G. A Time-Varying Complex Dynamical Network Model and Its Controlled Synchronization Criteria. IEEE T. Automat. Contr. 50, 841-846 (2005).

30. Motter, A. E., Zhou, C. S. \& Kurths, J. Enhancing complex-network synchronization. Europhys. Lett. 69, 334-340 (2005).

31. Acebrón, J. A., Bonilla, L. L., Vicente, C. J. P., Ritort, F. \& Spigler, R. The Kuramoto model: A simple paradigm for synchronization phenomena. Rev. Mod. Phys. 77, 137-185 (2005).

32. Osipov, G., Kurths, J. \& Zhou, C. Synchronization in oscillatory networks. (Springer Verlag, New York, 2007).

33. Arenas, A., Díaz-Guilera, A., Kurths, J., Moreno, Y. \& Zhou, C. Synchronization in complex networks. Phys. Rep. 469, 93-153 (2008).

34. Boccaletti, S. The synchronized dynamics of complex systems. (Elsevier, Amsterdam, 2008).

35. Barrat, A., Barthelemy, M. \& Vespignani, A. Dynamical Processes on Complex Networks. (Cambridge Univ. Press, Cambridge, 2008).

36. Louzada, V. H. P., Araújo, N. A. M., Andrade, J. S. \& Herrmann, H. J. How to suppress undesired synchronization. Sci. Rep. 2, 658 (2012).

37. Nicosia, V., Valencia, M., Chavez, M., Diaz-guilera, A. \& Latora, V. Remote synchronization reveals network symmetries and functional modules. Phys. Rev. Lett. 110, 174102 (2013)

38. Schuster, H. G. \& Wagner, P. Mutual Entrainment of Two Limit Cycle Oscillators with Time Delayed Coupling. Prog. Theor. Phys. 81, 939-945 (1989).

39. Yeung, M. K. \& Strogatz, S. H. Time Delay in the Kuramoto Model of Coupled Oscillators. Phys. Rev. Lett. 82, 648 (1999).

40. Choi, M. Y., Kim, H. J., Kim, D. \& Hong, H. Synchronization in a system of globally coupled oscillators with time delay. Phys. Rev. E 61, 371-381 (2000).
41. Dahms, T., Lehnert, J. \& Schöll, E. Cluster and group synchronization in delaycoupled networks. Phys. Rev. E 86, 016202 (2012)

42. Williams, C. R. S. et al. Experimental Observations of Group Synchrony in a System of Chaotic Optoelectronic Oscillators. Phys. Rev. Lett. 110, 064104 (2013)

43. Gallos, L. K., Makse, H. A. \& Sigman, M. A small world of weak ties provides optimal global integration of self-similar modules in functional brain networks. Proc. Nat. Acad. Sci. USA 109, 2825-2830 (2012).

44. Li, G. et al. Towards Design Principles for Optimal Transport Networks. Phys. Rev. Lett. 104, 018701 (2010)

45. Li, G. et al. Optimal transport exponent in spatially embedded networks. Phys. Rev. E 87, 042810 (2013).

46. Hodgkin, A. L. \& Huxley, A. F. A quantitative description of membrane current and its application to conduction and excitation in nerve. J. Physiol. 117, 500-544 (1952).

47. Shams, L., Kamitani, Y. \& Shimojo, S. Illusions: What you see is what you hear. Nature 408, 2000 (2000).

48. Hairston, W. D. et al. Visual localization ability influences cross-modal bias. J. Cogn. Neurosci. 15, 20-29 (2003).

\section{Acknowledgments}

Authors would like to thank the Swiss National Science Foundation under contract 200021 126853, the CNPq, Conselho Nacional de Desenvolvimento Científico e Tecnológico Brasil, the CNPq/FUNCAP Pronex grant, the ETH Zurich Risk Center, and the INCT-SC-Brasil for financial support. This work was also supported by grant number FP7-319968 of the European Research Council. We would like also to thank L. de Arcangelis, A. Gower, F. Mohseni, and K. J. Schrenk for the valuable discussions.

\section{Author contributions}

V.H.P.L., N.A.M.A., J.S.A. and H.J.H. wrote the main text, prepared the simulations, and discussed the results.

\section{Additional information}

Supplementary information accompanies this paper at http://www.nature.com/ scientificreports

Competing financial interests: The authors declare no competing financial interests. How to cite this article: Louzada, V.H.P., Araújo, N.A.M., Andrade, J.S. \& Herrmann, H.J Breathing synchronization in interconnected networks. Sci. Rep. 3, 3289; DOI:10.1038/ srep03289 (2013)

(c) (i) $(-)$ This work is licensed under a Creative Commons Attribution-

NonCommercial-NoDerivs 3.0 Unported license. To view a copy of this license visit http://creativecommons.org/licenses/by-nc-nd/3.0 